\title{
Galaxies and the intergalactic medium in groups
}

\author{
Trevor J. Ponman, Ed Lloyd-Davies \& Stephen F. Helsdon \\ School of Physics and Astronomy, University of Birmingham, \\ Edgbaston, Birmingham B15 2TT, UK
}

\begin{abstract}
The study of the relationship between the hot gas in groups and the galaxies they contain can help to constrain the evolution of both galaxies and groups. Here we present evidence that the intergalactic medium in groups has been strongly affected by preheating associated with galaxy formation which mostly preceded group collapse. The presence of this effect appears to be unrelated to the morphology of group galaxies, which supports models in which galaxy types are not primordial. We also present preliminary evidence that early-type galaxies in groups are not underluminous in the X-ray compared to isolated galaxies, suggesting that their dark halos may not have been substantially stripped.
\end{abstract}

\section{Introduction}

The discovery of the morphology-density relation for galaxies (i.e. the predominance of early-type galaxies in rich environments) led to a 'nature-nurture' debate: do galaxies have their morphology determined by the circumstances of their birth, or have their observed forms been determined primarily by subsequent environmentally sensitive processes? Posed in this way, the question now sounds a little old-fashioned, since it is clear that environmental effects, such as galaxy interactions and mergers, do have a significant effect on at least some galaxies. However, in many respects the nature-nurture issue is still with us, since it remains unclear whether most galaxies have undergone substantial morphological changes since their formation. Currently popular 'semi-analytic' galaxy formation models (e.g. Kauffmann et al 1993) involve repeated major morphological change in galaxies, as spirals merge to form ellipticals and ellipticals accrete discs to become spirals. On the other hand, most of the work on chemical evolution of clusters has been based on the premise that metals are released only from early-type galaxies, and that these are formed in an initial star-forming burst.

The aim of the present paper is to discuss some ways in which light may be shed on these questions by examining the properties of galaxies and of the intergalactic medium (IGM) within groups. Groups are more illuminating than clusters for such a study, since galaxies can have a stronger impact on the IGM in groups, and galaxy interactions are also likely to be stronger given the lower velocity dispersion. We look first at the evidence that energetic phenomena in galaxies have significantly affected the structure of the IGM in groups, and then 
at the X-ray properties of group galaxies, and their relationship with broader group properties.

\section{The impact of galaxies on the hot IGM}

Studies of starburst and merging galaxies in the local universe show (Heckman et al 1990, Read \& Ponman 1998) that such vigorous star-forming activity can power substantial outflows of hot gas from galaxies. Such energy injection will be more significant in groups, where the virial temperature is typically $\sim 1 \mathrm{keV}$, than in clusters. The result should be to break the self-similarity of the gas distribution in clusters of different mass. Ponman et al (1999) looked for such an effect by overlaying X-ray surface brightness profiles of systems covering a wide temperature range, each profile being scaled to allow for the larger size and higher brightness expected in hotter clusters on the basis of self-similar scaling. The result, shown in Fig.1, indicates that the X-ray brightness in systems with $T<4 \mathrm{keV}$ is suppressed below the values expected on the basis of a self-similar scaling from clusters. This is just the effect one expects if injection of galaxy winds has significantly raised the entropy of the gas (Cavaliere et al 1997).

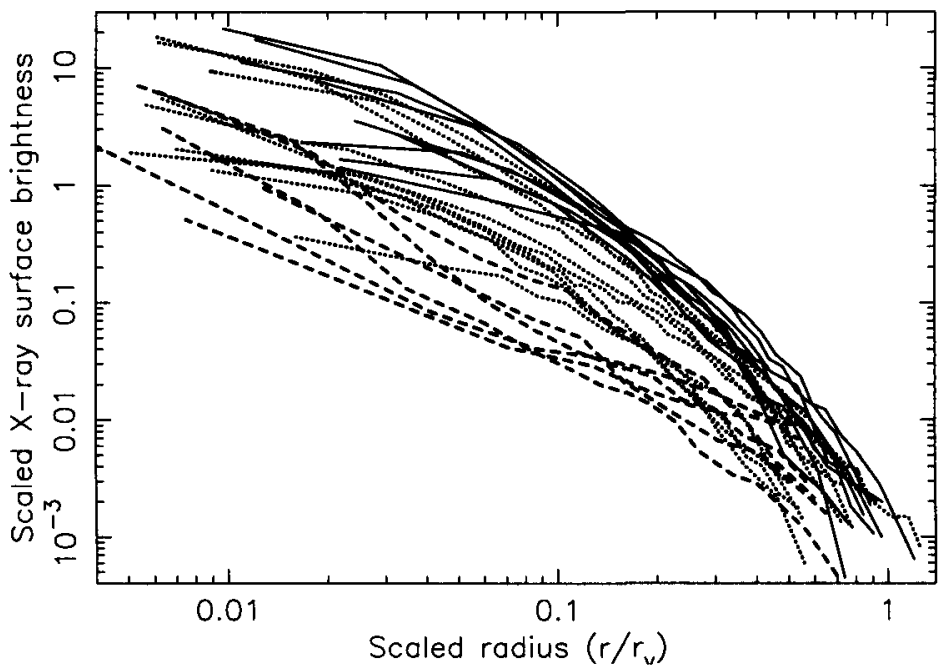

Figure 1. X-ray surface brightness profiles from the ROSAT PSPC for a set of 25 systems spanning a wide range in X-ray temperature have been scaled in radius and surface brightness according to the selfsimilar relations. Systems with $T>4 \mathrm{keV}$ are plotted as solid lines, $2<T<4 \mathrm{keV}$ as dotted, and $T<2 \mathrm{keV}$ as dashed. Outside any central cooling flow region, clusters with $T>4 \mathrm{keV}$ appear to be approximately self-similar, whilst cooler systems have systematically lower and flatter profiles. 
In order to investigate this further, we need to derive density and temperature distributions within the IGM, so that its entropy and energy can be determined. To do this, spherically symmetric models of the gas properties have been fitted to X-ray spectral images for a set of 20 systems, ranging in mean temperature from 0.5 to $14 \mathrm{keV}$. ROSAT PSPC data are used for all systems, but in the case of the hotter clusters, this is unable to constrain $T$ effectively, and ASCA GIS data have been fitted simultaneously with the ROSAT data in such cases. The fitting technique involves adjusting the parameters in simple analytic representations of the radial density and temperature distributions until a maximum likelihood fit is obtained. The effects of projection and PSF blurring are allowed for during this process. Details are given in Lloyd-Davies et al (1999).

From the best fit profiles for electron number density $n_{e}(r)$, and temperature $T(r)$, the gas 'entropy', $S=T / n_{e}^{2 / 3}$, can be derived. In the simple self-similar case, entropy at a given fraction of the virial radius should scale as $T^{-1}(1+$ $z)^{2}$. In Fig.2, we show the derived entropy profiles for our systems (grouped together into four temperature bins for clarity) scaled according to this selfsimilar prescription. The central regions (within 0.05-0.1 $R_{v}$ ) are affected by cooling, but at larger radii, where cooling times exceed the Hubble time, it is apparent that systems with $T<3.7 \mathrm{keV}$ have higher scaled entropies than hotter clusters.

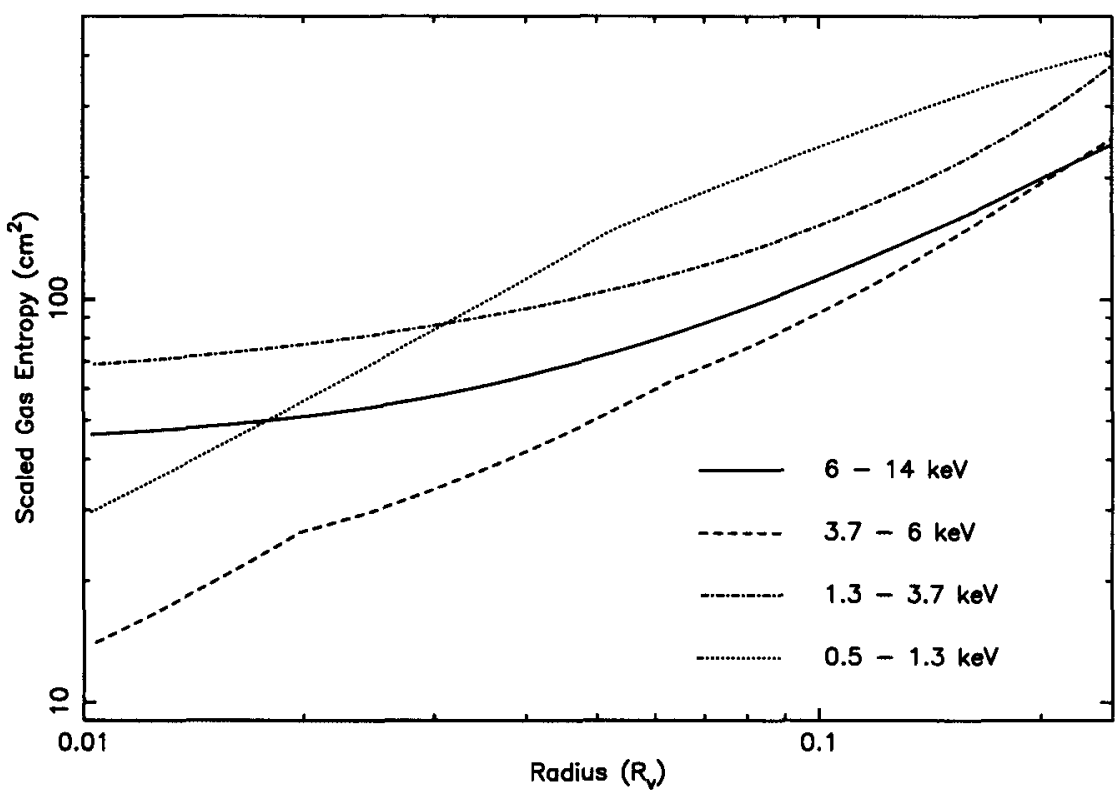

Figure 2. Scaled entropy profiles averaged over systems within four different temperature bands are plotted as a function of scaled radius $\left(r / R_{v}\right)$. Outside the central cooling region, scaled entropy is higher in cooler systems. 
The effect can be seen in more detail in Fig.3, where the unscaled entropy at a fiducial radius of $0.1 R_{v}$ is shown for each of the 20 systems, plotted against system temperature. Below $T \sim 3 \mathrm{keV}$, the entropy departs from the selfsimilar prediction (dotted line) and flattens off to an approximately constant value. A weighted mean for the four lowest temperature groups gives a value of $S_{0}=139 \pm 7 \mathrm{~h}_{50}^{-1 / 3} \mathrm{keV} \mathrm{cm}{ }^{2}$ for this floor entropy (where $\mathrm{h}_{50}$ is $H_{0} / 50 \mathrm{~km}$ $\mathrm{s}^{-1} \mathrm{Mpc}^{-1}$ ). Evaluating the excess entropy, above the self-similar value (i.e. above the dotted line in Fig.3) for the 12 systems with $T<4 \mathrm{keV}$, we obtain $\Delta S=68 \pm 12 \mathrm{keV} \mathrm{cm}{ }^{2}$.

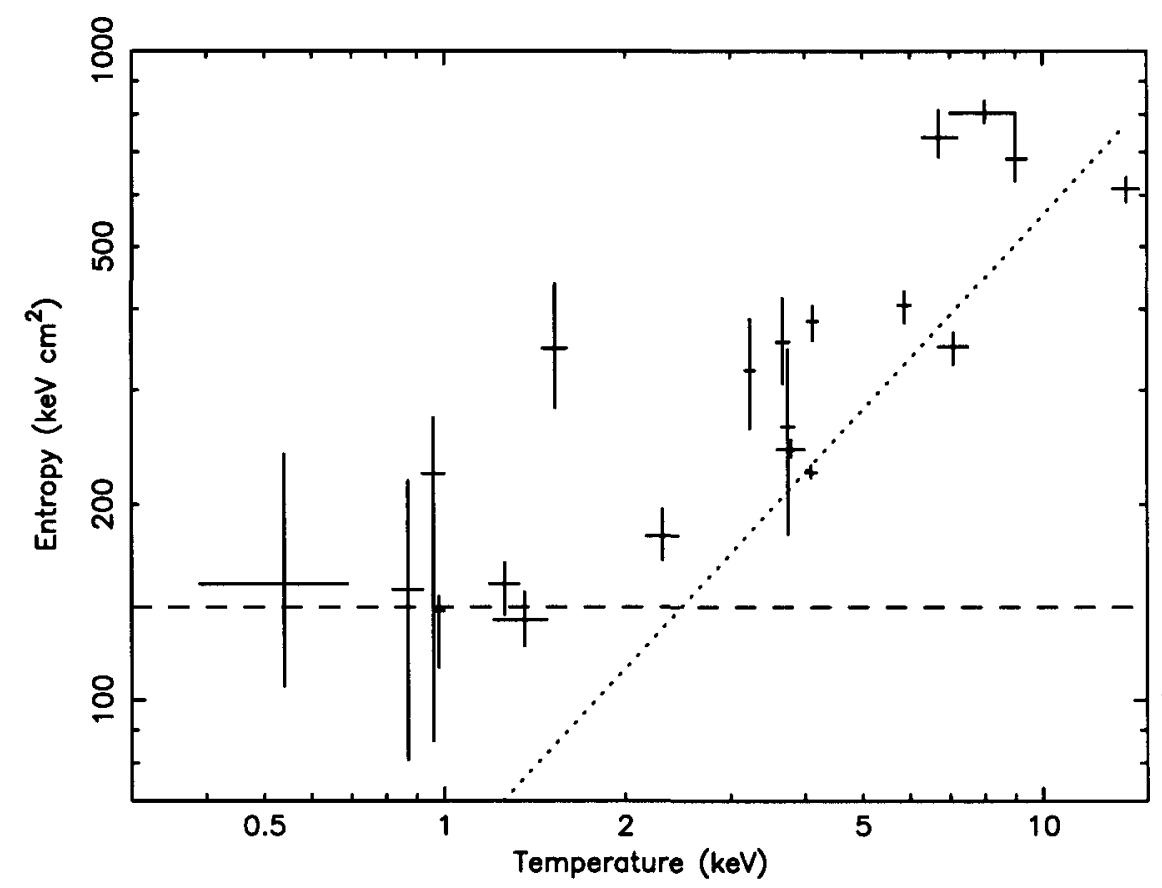

Figure 3. Gas entropy at $0.1 R_{v}$ plotted against mean temperature for 20 systems. The dotted line is a self-similar relation $(S \propto T)$ fitted to the systems above $4 \mathrm{keV}$, excluding $A 665$ and A2218, in which the entropy appears to have been raised by the effects of recent merging. The dashed line shows the derived floor entropy of $139 \mathrm{~h}_{50}^{-1 / 3} \mathrm{keV} \mathrm{cm}$.

We can also use our fitted models to investigate the binding energy $\left(E_{b}\right)$ of the IGM. Self-similarity predicts that this should scale with the depth of the potential, so that $E_{b} \propto T$. In Fig.4, we show the specific binding energy (i.e. thermal-gravitational energy) for the innermost gas in each system, plotted against mean system temperature. It is important to compare the equivalent mass of gas in each case, and we have integrated over a gas mass corresponding to $0.4 \%$ of the virial mass (derived from the expression of Navarro et al 1995) in each system. This typically corresponds to the gas within $0.1-0.2 R_{v}$. Comparing the data with the self-similar relation (dashed line), it can be seen that 
cooler systems have a reduced gas binding energy. If energy has been injected from galaxy winds, then the amount of this excess energy energy might be expected to scale with the galaxy mass. Taking this to be a fixed fraction of the (unperturbed) gas mass, we arrive at a simple model for the binding energy $E_{b}=A T-\Delta E$, where $\Delta E$ is the injected energy per particle. The solid line in Fig.4 shows the result of a weighted best fit of such a relationship to the data - giving a best fit excess energy of $\Delta E=0.44 \mathrm{keV}$. We have excluded the cluster $\mathrm{A} 400(T=2.3 \mathrm{keV})$ from this fit. This cluster has an anomalously low entropy, with a small statistical error, which is almost certainly spurious, since a dynamical study by Beers et al (1992) shows that the cluster probably has two major components superposed along the line of sight. Inclusion of A400 in the fit gives the result shown in Fig.4 by the dot-dash line, which is a poor fit to the cooler systems.

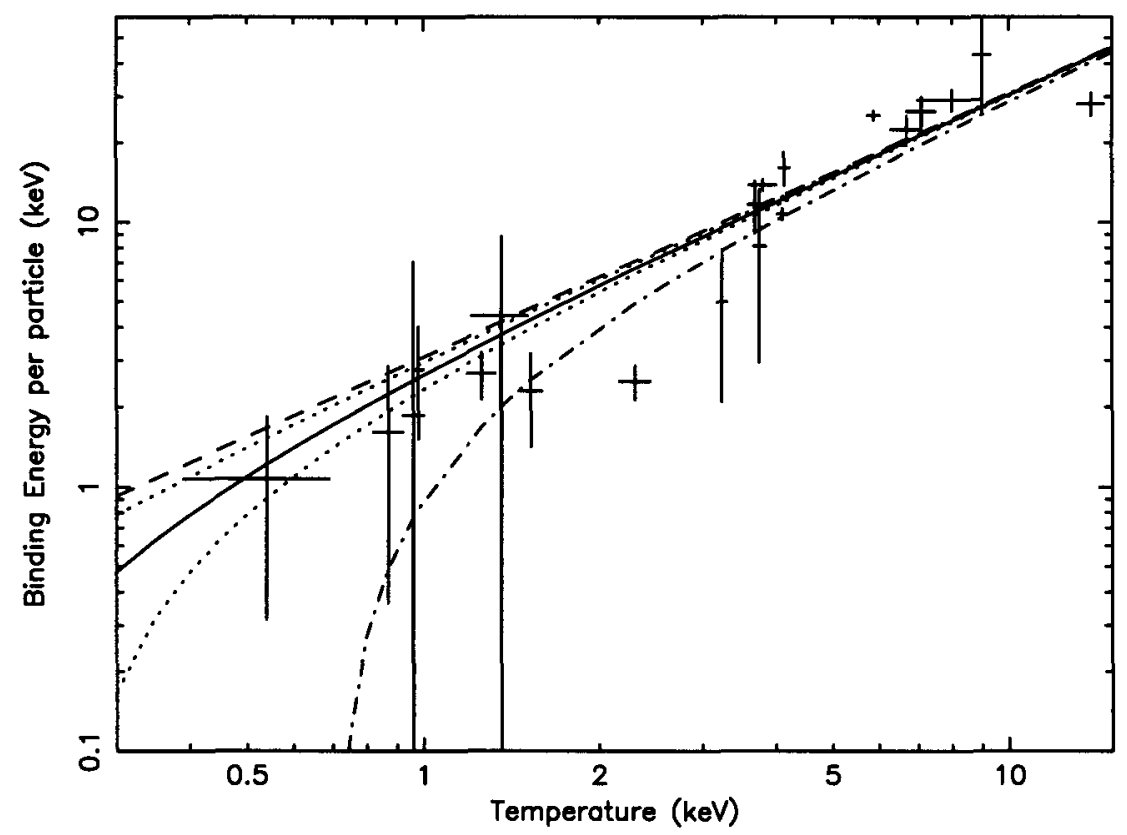

Figure 4. Mean binding energy per particle of the central $0.004 M_{v}$ of gas plotted against mean system temperature. The dashed line shows an $E_{b} \propto T$ fit to systems with $T>4 \mathrm{keV}$. The solid line with dotted $1 \sigma$ confidence region, shows our best fit relation, involving $\Delta E=0.44 \mathrm{keV}$ per particle of excess energy.

The excess energy of $0.44 \mathrm{keV}$ corresponds to a temperature rise of $0.3 \mathrm{keV}$. Combining this value with the observed excess entropy $\left(68 \mathrm{keV} \mathrm{cm}^{2}\right)$, and noting that $\Delta S=\Delta T / n_{e}^{2 / 3}$, we can derive a value for the density of the gas at the time of energy injection. The resulting value, $n_{e}=3 \times 10^{-4} \mathrm{~h}_{50}^{1 / 2} \mathrm{~cm}^{-3}$, is well below the gas density seen in cluster cores at the present time. This implies that the energy must have been injected at an epoch before clusters were fully formed, but 
after the epoch $(z \sim 10)$ at which the mean gas density in the universe dropped to $3 \times 10^{-4} \mathrm{~cm}^{-3}$. In other words, these results support the idea (Kaiser 1991) that the gas was preheated at an epoch before cluster formation, presumably as a result of energetic winds accompanying galaxy formation. Such a scenario is also able to explain the lack of strong X-ray luminosity evolution observed in clusters (Bower 1997).

A quite separate indication of the extent to which galaxy ejecta have affected the IGM can be obtained from the study of the heavy element distribution in groups and clusters. It is encouraging that recent studies with ASCA, which can map both $\mathrm{Fe}$ and $\mathrm{Si}$, and hence separate the effects of type Ia and type II supernovae, give results which agree very well with preheating by $\Delta E \sim 0.4 \mathrm{keV}$, provided that most of the supernova energy is able to escape from galaxies during massive bursts of star formation (Finoguenov \& Ponman 1999).

\section{Galaxies in groups}

The properties of galaxies may be affected by the group environment. A number of examples of such effects have been noted, especially in compact groups where galaxy interactions can trigger star formation and lead to morphological distortions or gas loss in late type galaxies (Hickson 1997). The properties of early type galaxies in groups has received less attention, and here we consider their X-ray properties.

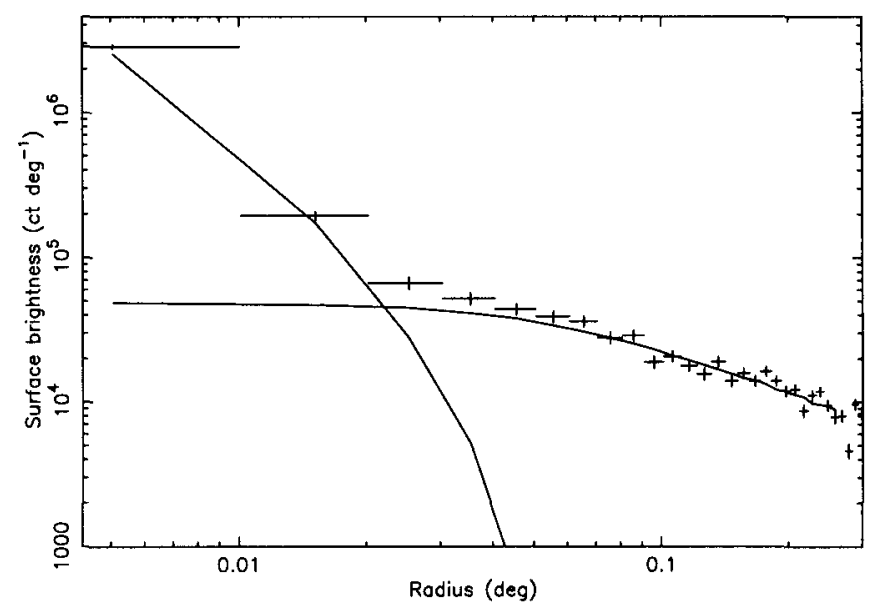

Figure 5. ROSAT PSPC surface brightness profile of the NGC4261 group, with best fitting two-component model King model overlaid. 
It is well known that the X-ray luminosity of early-type galaxies shows a very wide spread, even at a given optical luminosity. It has been suggested that this may be due to the influence of environment on the properties of central galaxies (Brighenti et al 1998). It is clear that many 'field ellipticals' are actually in groups, however no systematic study of the extent to which $L_{X} / L_{B}$ differs in group compared to isolated ellipticals has yet been carried out.

Helsdon \& Ponman (1999) have studied the X-ray properties of a sample of $24 \mathrm{X}$-ray bright loose groups in detail. In such systems it is difficult to separate the emission from central galaxies from that in the group. However, following Mulchaey \& Zabludoff (1998), we find that two components are required to adequately fit the profiles of most systems. An example is shown in Fig.5. Identifying the central component with the galaxy itself, and the more extended component with the group, we are able to derive $L_{X}$ even for these central galaxies. For galaxies elsewhere in groups it is generally much easier to derive the luminosity associated with the galaxy.

One interesting feature of $\mathrm{X}$-ray bright groups is that the brightest group galaxy (BGG) is almost invariably of early type. In Fig.6 we show how the X-ray luminosities we derive for BGGs correlate with their optical luminosity, and with the value of $L_{X}$ for the group as a whole. The much stronger correlation seen in the right hand plot indicates that the central component should be identified with the group itself, rather than just the central galaxy. In most of the systems with such central X-ray components, we also see evidence in the PSPC data for central cooling, which strongly suggests that these components are group cooling flows, rather than normal hot galaxy halos.
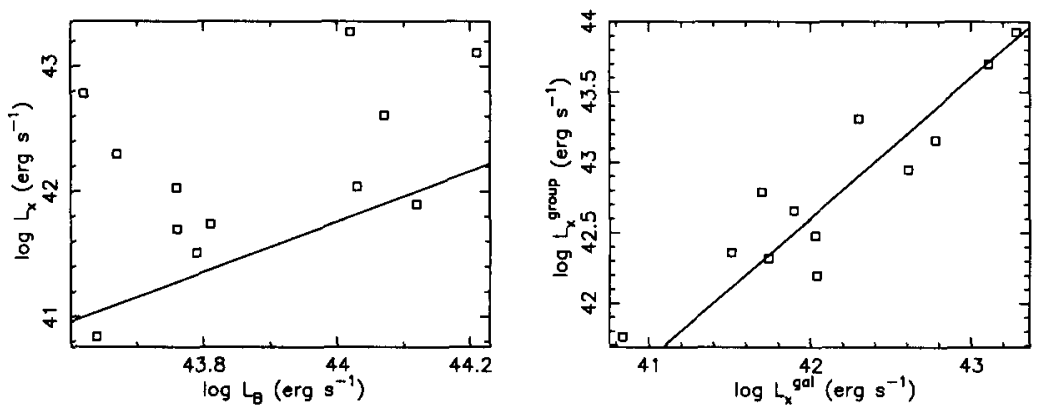

Figure 6. These plots show the relationship between the X-ray luminosity of the BGG and (left) its optical luminosity, and (right) the $\mathrm{X}$-ray luminosity of the group as a whole. The lines overplotted are (left) the $L_{X}: L_{B}$ relation derived for elliptical galaxies by Eskridge et al (1995), and (right) the line corresponding to $L_{B G G}=0.25 L_{\text {group }}$. 
Combining the results for all early-type galaxies in the loose group sample (both central and non-central galaxies) and adding luminosities derived from an HRI study of a sample of compact galaxy groups (Helsdon et al, in preparation), we show in Fig.7 the relationship between X-ray and optical luminosity for his combined group galaxy sample.

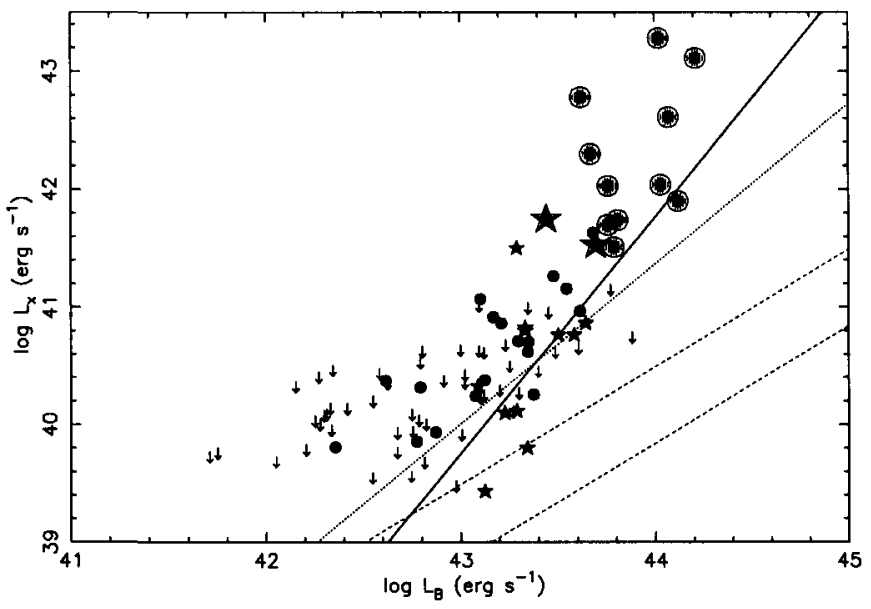

Figure 7. X-ray:optical luminosity relation for a combined sample of early type galaxies in loose (circles) and compact (stars) galaxy groups. Two sigma upper limits are marked with down-arrows. The galaxies plotted with large symbols are the dominant galaxies in their respective groups, and their X-ray emission is probably dominated in most cases by a group cooling flow. The solid line shows the relation derived for field galaxies by Eskridge et al (1995), the dotted line shows the corresponding relation for field spirals, and the dashed lines show two different estimates of the discrete source contribution to $L_{X}$ which are consistent with the survey of Beuing et al (1999).

The first point which is clear is that all the most luminous galaxies are BGGS. This strongly suggests that contamination by group emission is responsible for most of the upper envelope in the $L_{X} / L_{B}$ relation for early-type galaxies. A less detailed analysis, which simply included all $\mathrm{X}$-ray emission around such galaxies, would yield even higher values of $L_{X} / L_{B}$ for such galaxies. Secondly, even once the BGGs are excluded, it appears that group galaxies are not underluminous compared to the field relation - though a proper statistical analysis including the upper limits is required to confirm this preliminary result. This conclusion is of considerable interest, since one might expect that the effect of galaxy interaction in groups would be to strip much of the extensive dark halos inferred to be present in field ellipticals. This in turn should lead to smaller, less 
luminous hot gas halos. Hence our result suggests that dark halo stripping is not very effective within groups, a surprising result which would have substantial implications for the dynamical evolution of groups.

One immediate concern about this result is the status of the 'field' relation plotted in the figure. Could this already be seriously affected by the presence of group galaxies within field samples? To test this, we derived an isolated elliptical subsample from the ROSAT sample of Beuing et al 1999, by removing all galaxies listed in cluster and group catalogues. The $L_{X}: L_{B}$ relation derived from this isolated subsample was found to be almost identical to that of Eskridge et al (1995).

Finally, we examine the relationship between the morphology of group galaxies and the shape of a group's surface brightness profile. If, as has been commonly assumed, most energy and metals are injected into the IGM by early type galaxies, we would expect systems with low spiral fraction to show flatter surface brightness profiles, and hence lower values of $\beta_{\text {fit }}$. (Surface brightness is parameterised in the conventional fashion as

$$
S(r)=S_{0}\left(1+r^{2} / r_{c}^{2}\right)^{-3 \beta_{\mathrm{fit}}+1 / 2} \text {.) }
$$

In practice, Fig. 8 shows no trend in this direction, especially when only the more reliable points (circled) are considered. This provides considerable support for hierarchical merger models, in which the distinction between early and late type galaxies is not fundamental.

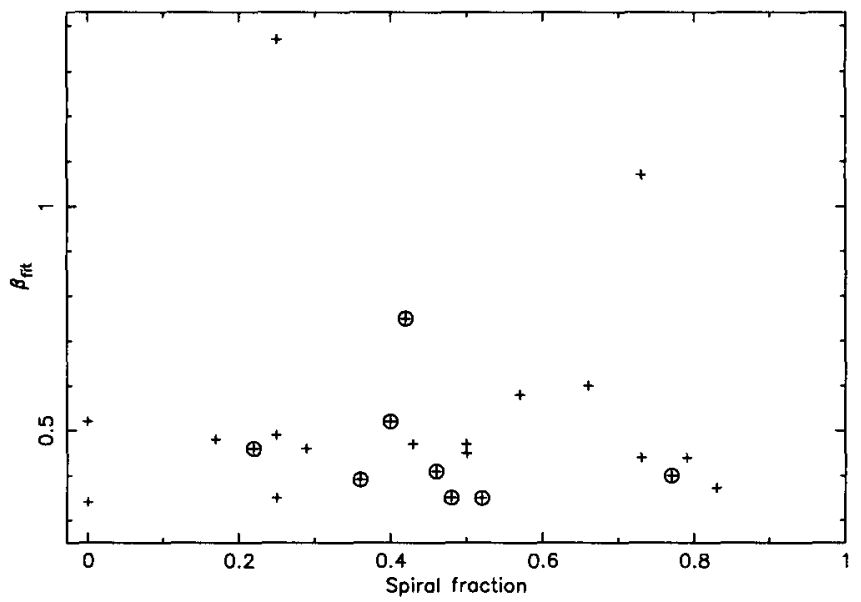

Figure 8. Relationship between spiral fraction and surface brightness index $\beta_{\text {fit }}$ for the loose group sample. Circled points denote the systems with the best determined surface brightness profiles. No trend is seen towards flatter profiles in systems containing many early-type galaxies. 
Acknowledgments. We would like to thank Damian Cannon, Julio Navarro and Ewan O'Sullivan for their contributions to this work.

\section{References}

Beers T. C. et al, 1992, ApJ, 400, 410

Beuing J. et al, 1999, MNRAS, 302, 209

Bower R. G., 1997, MNRAS, 288, 355

Brighenti F,. Matthews W. G., 1998, ApJ, 495, 239

Cavaliere A., Menci N., Tozzi P., 1997, ApJ, 484, L21

Eskridge P. B., Fabbiano G., Kim D., 1995, ApJS, 97, 141

Finoguenov A., Ponman T.J., 1999, MNRAS, 305, 325

Heckman T. M., Armus L. M., Miley G. K., 1990, ApJS, 74, 833

Helsdon S. F., Ponman T. J., 1999, MNRAS, submitted

Hickson P., 1997, ARAA, 35, 357

Kaiser N., 1991, ApJ, 383, 104

Kauffmann G., White S. D. M., Guideroni B., 1993, MNRAS, 264, 201

Lloyd-Davies E. J., Ponman T. J., Cannon D. B., 1999, MNRAS, submitted Mulchaey J.S., Zabludoff A.I., 1998, ApJ, 496, 73

Navarro J. F., Frenk C. S., White S. D. M., 1995, MNRAS, 275, 720

Ponman T. J., Cannon D. B., Navarro J. F., 1999, Nature, 397, 135

Read A. M., Ponman T. J., 1998, MNRAS, 297, 143 